\title{
Position Control for the Spherical Joint Driven by PMAs
}

\author{
Qing-shan Huang \\ School of Automation \\ BeiJing Institute of Technology \\ BeiJing, China,13811024073 \\ 443555987@163.com
}

\author{
Jin-bing Chen \\ School of Automation \\ BeiJing Institute of Technology \\ BeiJing, China,13801284212 \\ smcchen@126.com
}

\begin{abstract}
A spherical joint system driven by pneumatic artificial muscle is constructed. As pneumatic artificial muscle has strong nonlinear characteristics and traditional control methods are hard to overcome the contradictions among control precision, adjusting speed and stability, the 2 order auto-disturbancerejection controller is introduced. The position control of the spherical joint can be realized by auto-disturbance-rejection controller or PID controller. The experimental results show that auto-disturbance-rejection controller can achieve better control effect than PID controller with steady precision less than $0.4^{\circ}$.
\end{abstract}

Keywords-PMA;spherical joint; position control;ADRC

\section{INTRODUCTION}

Joint driving system is an important part of the robot. In order to construct robot joints which have large output torque, simple structure, large sports range and easy control, domestic and foreign experts and scholars have done research, and made a series of achievements. For example, paper [1] designs a new type of three-degree-of freedom vertical fellowship movement decoupling hydraulic servo joint, which has highenergy density and can be applicable to the robot arm operation, paper [2] designs a high-performance robot joint integrated drive system by using directly drive technology for ac servo system.

As a new type of pneumatic actuator, pneumatic artificial muscle responds quickly and moves smoothly. It can achieve very slow motion, which is close to the natural biological movement. As a result, it generates low heat and noise in the operating process ${ }^{[3]}$.Compared with traditional actuators (motor, air cylinder), it has many advantages, such as simple structure, great flexibility, high output force/weight, etc. ${ }^{[4]}$. So the pneumatic artificial muscle is suitable as the drive of robot joints.

This paper puts forward a spherical joint system driven by pneumatic artificial muscle. Pneumatic artificial muscle has very strong nonlinear characteristics ${ }^{[5]}$, so the autodisturbance-rejection controller is introduced to realize control. As the improvement on PID, ADRC eliminates integral link and adds the extended state observer which can realize realtime estimation for the internal model perturbation and external disturbance. It retains the advantages of the PID controller, and overcomes the defects of lower controlling precision by using nonlinear error state feedback strategy ${ }^{[6]}$. ADRC can achieve good control effect when it is applied to the system.

\section{SPHERICAL JOINT SYSTEM}

The spherical joint position control system, which takes industrial PC as the core, is made up of spherical joint mechanical bench, pneumatic circuits, data acquisition and control system. The principle diagram is shown in figure 1 .

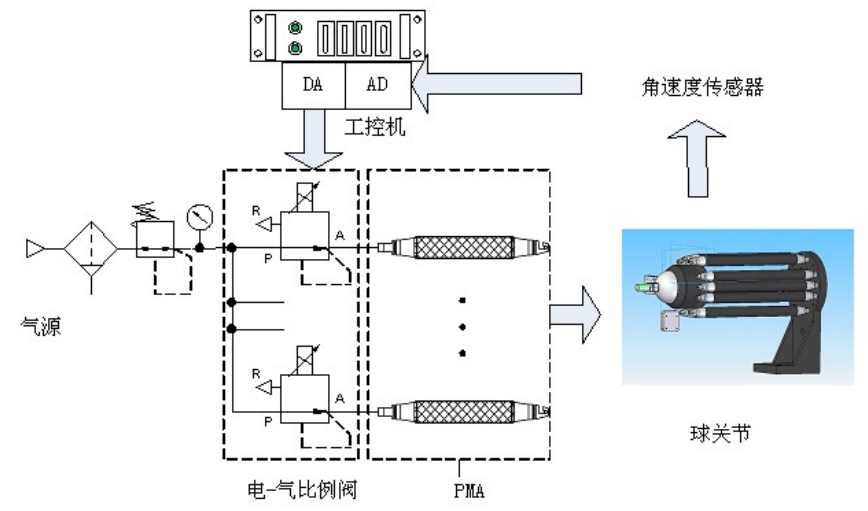

Figure 1.the principle diagram of spherical joint position control system

The spherical joint mechanical bench consists of orthogonal supports, cylindrical body connection and sphere. Pneumatic artificial muscle is distributed in the body connection, the axis of two angular velocity sensor overlaps the axis of spherical. It is shown in figure 2.

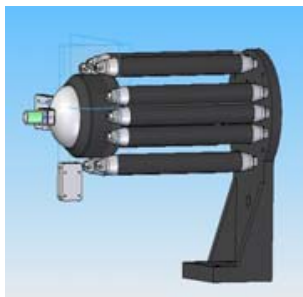

(1)schematic diagram

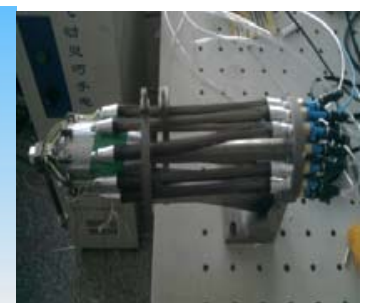

(2)physical figure
Figure 2.spherical joint mechanical bench

Pneumatic circuits consist of the air supply, pneumatic triple, electric proportional valve and pneumatic artificial 
muscle. Data acquisition and control system realizes sensor data acquisition and processing as well as the motions of joint control output by using data acquisition card PCI-818, PCI727.

TABLE I. Components Parameters

\begin{tabular}{|c|c|c|}
\hline & Name & Technology parameters \\
\hline 1 & $\begin{array}{c}\text { electric } \\
\text { proportional } \\
\text { valve }\end{array}$ & ITV0050 0-0.9MPa \\
\hline 2 & $\begin{array}{c}\text { angular velocity } \\
\text { sensor }\end{array}$ & $\begin{array}{c}\text { LCG50-00250-100 0-5V } \\
\text { sensitivity } 6.4 \mathrm{mV} / \% \text { sec }\end{array}$ \\
\hline 3 & $\begin{array}{c}\text { data acquisition } \\
\text { card }\end{array}$ & $\begin{array}{c}\text { PCI-818 } 16 \text { road one-port analogue } \\
\text { inputs } \\
\text { PCI-727 12 road simulated output }\end{array}$ \\
\hline
\end{tabular}

\section{ADRC DESIGN}

It is hard to build up the nonlinear model of the pneumatic artificial muscle, while ADRC can treat the uncertainty of the model as the system disturbance and give compensation by ESO that estimates the real time action, so one ADRC controller is designed to realize control. ADRC consists of tracking differentiator, extended state observer and nonlinear state error feedback ${ }^{[7]}$.Its structure is shown in figure 3 below.

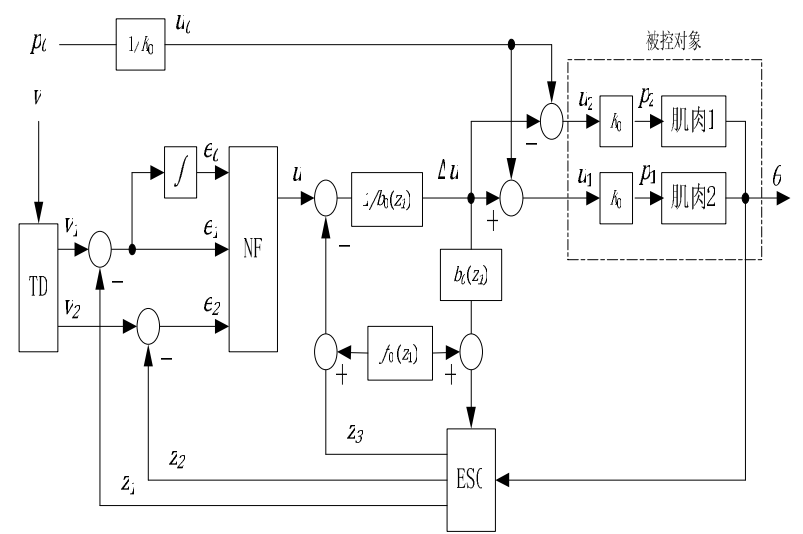

Figure 3. ADRC structure chart

Tracking differentiator can quickly track input signals without overshoot or flutter. It can increase the signal-to-noise ratio and give better-quality differential signals. The discrete form is expressed by equation (1) (2) (3), which is formed by the steepest comprehensive function for 2 order discrete system.

$$
\begin{aligned}
& f_{h}=\operatorname{fhan}\left(v_{1}(k)-v(k), v_{2}(k), r_{0}, h_{0}\right) \\
& v_{2}(k+1)=v_{2}(k)+h f_{h} \\
& v_{1}(k+1)=v_{1}(k)+h v_{2}(k)
\end{aligned}
$$

Where, $\mathrm{h}$ is sampling time, $r_{0}$ is speed factor, $h_{0}$ is filter factor.

Extended state observer that estimates the real time action can give compensation to simplify control object. 3 order extended state observer is expressed by equation (4) (5) (6) (7) (8) (9).

$$
\begin{aligned}
& e=z_{1}(k)-y(k) \\
& f_{e}=\operatorname{fal}\left(e, 0.5, h_{1}\right) \\
& f_{e 1}=\operatorname{fal}\left(e, 0.25, h_{1}\right) \\
& z_{1}(k+1)=z_{1}(k)+h\left(z_{2}(k)-\beta_{01} e\right) \\
& z_{2}(k+1)=z_{2}(k)+h\left(z_{3}(k)-\beta_{02} f_{e}\right. \\
& \left.\left.+f_{0}\left(z_{1}(k)\right)+b_{0}\left(z_{1}(k)\right) \Delta u(k)\right)\right) \\
& z_{3}(k+1)=z_{3}(k)+h\left(-\beta_{03} f_{e 1}\right)
\end{aligned}
$$

Where, $z_{1}, z_{2}, z_{3}$ track $\mathrm{x} 1, \mathrm{x} 2$, disturbance, $h_{1}$ is interval length of linear period , $\beta_{01}, \beta_{02}, \beta_{03}$ are observer parameters, $f_{0}=0, b_{0}=5000$.

Nonlinear state error feedback uses the smooth feedback to make the steady-state error diminish in index form and so accelerate response speed. It is expressed by equation (10) (11) (12) (13).

$$
\begin{aligned}
& e_{0}(k)=e_{0}(k-1)+e_{1}(k) \\
& e_{1}(k)=v_{1}(k)-z_{1}(k) \\
& e_{2}(k)=v_{2}(k)-z_{2}(k) \\
& u(k)=\beta_{0} e_{0}(k)+\beta_{1} \operatorname{fal}\left(e_{1}(k), 0.5, h_{2}\right) \\
& +\beta_{2} \operatorname{fal}\left(e_{2}(k), 1.5, h_{2}\right)
\end{aligned}
$$

Where, $h_{2}$ is estimated coefficient of linear period, $\beta_{0}$ 、 $\beta_{1} 、 \beta_{2}$ respectively are integral, scale, differential coefficient.

Finally $Z_{3}$ is compensated to control quantity to improve the control performance. It is expressed by equation (14).

$$
\Delta u=\frac{u(k)-z_{3}(k)-f_{0}\left(z_{1}(k)\right)}{b_{0}\left(z_{1}(k)\right)}
$$

\section{Single Freedom Degree Position Control}

The initial pressure of pneumatic artificial muscle is 0.25 MPa. The spherical joint system turns $20^{\circ}, 40^{\circ}, 60^{\circ}$ in single freedom degree by using ADRC and PID controllers. The step response is shown in figure 4.Controller parameters are as follows:

ADRC : $\mathrm{h}=0.01, \quad h_{0}=0.02, r_{0}=5800, h_{1}=$ 0.07, $\beta_{01}=80, \beta_{02}=1400, \beta_{03}=2800, h_{2}=0.11, \beta_{0}=0.2, \beta_{1}$ $=130, \beta_{2}=2$,

PID: $\quad k_{p}=1.1, k_{i}=0.05, k_{d}=0, \quad\left(20^{\circ}\right)$

$k_{p}=1.1, k_{i}=0.05, k_{d}=0,\left(40^{\circ}\right)$

$k_{p}=1.1, k_{i}=0.05, k_{d}=0, \quad\left(60^{\circ}\right)$ 


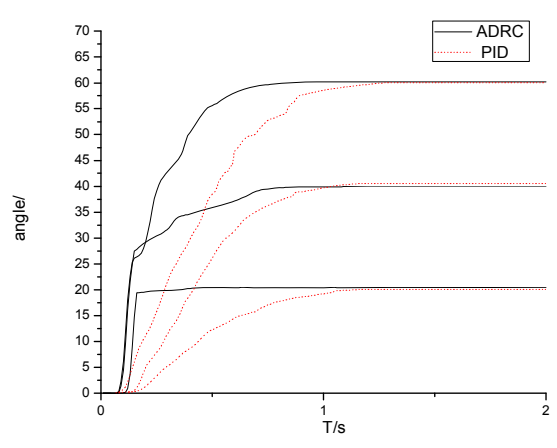

Figure 4.The step response comparison between ADRC and PID

As shown in Figure 4, the average steady-state error is less than $0.3^{\circ}$, and the average transition process time is about $0.6 \mathrm{~s}$ under the control of ADRC, while the average steady-state error is less than $0.4^{\circ}$, and the average transition process time is about 1s under the control of PID. Compared with PID, the average steady-state error reduces by $25 \%$ and the response time reduces by $40 \%$ adopting ADRC.

\section{V.Two FreEdOM DEGREE COMPOUND POSITION CONTROL}

The spherical joint position control target is represented by attitude matrix $T$,The included angles between corresponding coordinate system axis $\mathrm{X}, \mathrm{Y}, \mathrm{Z}$ and fixed coordinate system are $\alpha, \beta, \lambda$. The included angles $\alpha, \beta, \lambda$ only determine attitude matrix $T$.The system realizes the control target by ADRC. The control targets are $\alpha=8^{\circ}, \beta=6^{\circ}, \lambda=6^{\circ}$ and $\alpha=24^{\circ}, \beta=17^{\circ}, \lambda=17^{\circ}$. Controller parameters are as follows:

ADRC $1: \mathrm{h}=0.05, h_{0}=0.025, r_{0}=6200, h_{1}=$ 0.08, $\beta_{01}=70, \beta_{02}=1350, \beta_{03}=2650, h_{2}=0.14, \beta_{0}=0.34, \beta_{1}$ $=190, \beta_{2}=3.4$,

ADRC $2: \mathrm{h}=0.05, h_{0}=0.023, r_{0}=6100, h_{1}=$ 0.075, $\beta_{01}=80, \beta_{02}=1450, \beta_{03}=2700, h_{2}=0.13, \beta_{0}=0.3, \beta_{1}$ $=170, \beta_{2}=2.5$,

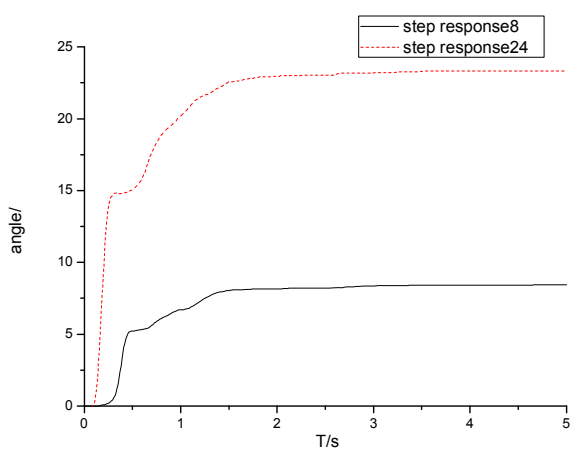

Figure 5. included angle of coordinate system axis $\mathrm{x}$

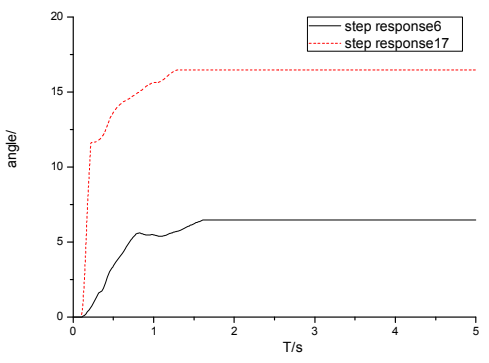

Figure 6. included angle of coordinate system axis y

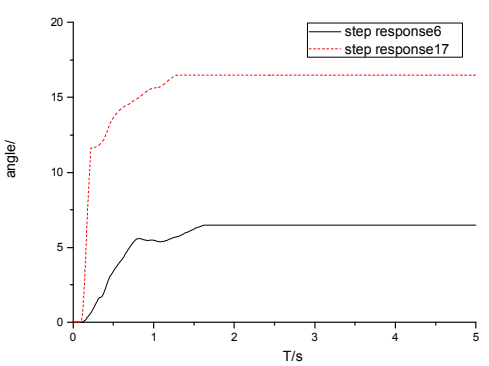

Figure 7. included angle of coordinate system axis z

As shown in Figure 5, 6 and 7, the steady-state time which reaches the target location is about 2 s, the steady-state error is less than $0.5^{\circ}$.It is shown that ADRC can achieve good spherical joint position control.

\section{VI.CONCLUSIONS}

As a new type of pneumatic actuator, pneumatic artificial muscle is taken seriously with good flexibility, but it has some disadvantages, such as linearity error, slow response speed and low repetition accuracy. In order to overcome the above shortcomings, this study adopts ADRC on spherical joint control experimental research and obtains ideal control effect that lays the foundation for multiple joint control.

\section{REFERENCES}

[1] Xin Long, Zhou Jiping,and Yan Jinping, "New Vertical Fellowship Movement Decoupling Hydraulic Servo Joint Design," China Mechanical Engineering, vol. A13, pp. 1824$1827,2002$.

[2] Wang Hong, Xu Dianguo,and Shi Jinzhuo, "Robot Joint Drive System,” High Technology Letters, vol. A14, pp. 47-50, 2004.

[3] Sui Liming, Bao Gang,and Wang Zu-wen, "Study on the characteristics of joint actuating by pneumatic artificial Muscle," Chinese Hydraulics \& Pneumatics, vol. A3, pp. 3-5, 2002.

[4] Fan Wei, Peng Guangzheng, and Huang Yu, "Pneumatic Muscle Actuator,” Machine Tool \& Hydraulics, vol. A1, pp. 32-36, 2003.

[5] ZHANG Hongli,SHEN Minmin,and PENG Guangzheng, "Static Mathematical Model and Experimental Study of Pneumatic Muscle Actuator," Chinese Hydraulics \& Pneumatics, vol. A4, pp. 17-19, 2009.

[6] Han Jinqin, "From PID technology to "auto-disturbance-rejection control" technology," Control Engineering of China, vol. A9, pp. 13-18, 2002.

[7] Han Jinqin, "Auto-disturbances-rejection controller and its application," Control and Decision, vol. A13, pp. 19—23, 1998. 